\title{
PAIN IN THE HEAD IN CONNECTION WITH CEREBRAL DISEASE.
}

\author{
BY DAVID FERRIER, M.D., F.R.B.
}

Ir seems at first sight strange, not to say unacconntable, that the centres of sensation und perception are themselves devoid of sensibility or sensitivity, and may be cut, lacerated, burnt, or otherwise injured, without the slightest indication of locel suffering being manifested. That this is true of the brain substance is 80 generally accepted on the evidence both of human pathology and experimental physiology, that it is scarcely necessary to argue the point. I have myself frequently verified it in the case of monkeys and other animals. After exposure of the brain, I have found that injuries which would cause acute pain if inflicted on other tissues and organs might be inflicted on the brain substance in an animal in full possession of conscionsness, and free to express feeling by any of its usual modes of manifestation, without exciting any signs of painful sensation.

When, however, we examine the conditions of sensitivity in tissues and organs which we know to be sensitive, the insensibility of the brain substance presents nothing extraordinary. Tissues and organs which are endowed with sensibility, whether in normal or abnormal conditions, are so in virtne of certain peripheral end-organs or nerve terminations, capable of being affected by what are termed stimuli, general or special. These organs are connected with a nerve centro by means of a nerve trunk of greater or less extent. Without this triple combination, the conditions of ensitivity do not exist, and we cannot speak of the various parts of the. apparatus as sensory except in relation to each other. Hence, 
apart, they have only the general properties of nerve tissues, bat they have no functions capable of being designated as sensory. Strictly speaking, we should not speak of sensory and motor nerves and nerve centres, but of sensory and motor apparatus:- the sensory being peripheral organ, nerve and centre, the motor being nerve cell, nerve and muscle.

The function depends on the collocation, as Lewes puts it.

The brain substance not being supplied with nerves and peripheral end-organs, injuries of the brain tissue cannot be felt as local pain, but will be manifested in disorders of the functions of the apparatus of which they form a part. Disease of a nerve centre which forms part of a muscular apparatus will show itself not at the seat of the lesion, but at the point where functional manifestation takes place. Hence it is a disorder of motility.

Disease of a nerve centre which is in relation with a peripheral receptive organ will be manifested as a disorder of receptivity or sensibility, not at the seat of lesion, but at the periphery where the end-organs are distributed.

Even when the peripheral organs no longer exist, diseases of those parts of the sensory apparatus still remaining are referred to the periphery with which they were formerly connected. In consequence of the organic memory of the nerve centres, functional excitations are projected peripherically, a fact which shows that the sensory apparatus must be regarded as a whole.

The brain centres being in relation either with muscular apparatus or peripherical sense organs, and being, further, the sulsstrata of subjective or mental manifestations, diseases of the brain may manifest themselves either in mental derangement, or in affections of motility and sensibility, or both; but as the brain tissures are devoid of nerves, they are devoid of sensibility, and cannot express their morbid states in locil pain.

We know, however, that certain diseases of the brain, in addition to the other indications of their presence, are accompanied with the most excruciating pain referred locally.

It would be very unsafe to diagnose cerebral disease from the character of the headnche alone, and it would probably 
be erroneous to refer headache in all cases of cerebral disease to an intracranial seat. But the headache in many forms of cerebral disease is of such a character, so allabsorbing in its intensity, differing much from all other forms of headache, sympathetic, neuralgic, \&c., and in particular because it frequently exactly corresponds with the seat of the disease and may be intensified by local irritation, that we may regard it as certain that the pain is intracranital, and that therefore the brain may indicate its disease by local suffering.

If it is true, as above laid down, that the brain substance is devoid of sensibility, and cannot under any conditions become sensitive, the seat of pain in cerebral disease must be sought elsewhere than in the brain tissue itself. It has, however, been advanced by Andral and others that though the brain is not sensitive under ordinary conditions, yet, like membranous structures which ordinarily possess little or no sensibility, it may become sensitive in morbid conditions such as inflammation. But there is no analogy between the two. For membranous structures which, under morbid conditions, may become the seat of acute pain are not devoid of nerves, while the brain substance is absolutely 80 .

But though the brain substance is devoid of nerves, it is invested and enclosed by membranons envelopes, and the view which has commended itself to many writers, both on theoretical grounds and from a consideration of the painful forms of cerebral disease, is that the seat of pain is in the cerebral membranes, particularly the dura mater.

That the dura mater is sensitive is a fact which, though at one time much contested, may now be regarded as firmly established. There may be Bome doubt as to the exact origin of the nerves which are distributed to the dura mater, but it is tolerably certain that they aro mainly derived from the fifth.

Luschka regarded these nerves as purely yascular; but Alexander ('Archiv für mik. Anat.' 1857) has demonstrated their distribution in the substance of this membrane.

It has likewise been demonstrated experimentally that certain forms of irritation, such as pinching, tearing and 
inflammatory conditions, give rise to indications of acute suffering in the lower animals. I have observed this especially in the comparatively thin dura mater of some of these. Duret, in a former number of this Joumal ('BrAIN,' Part I.), has called attention to the important purt which irritation of the nerves of the dura mater plays in traumatic cerebral lesions.

The sensibility of the dura mater is of much the snme character as that of fibrous membranes generally, of which it was at one time supposed to be the origin or mother. This is not perticularly obvious except under conditions of abnormal tension, wrenching or inflammation. In consequence of the comparatively sparse distribution of nerves, membranes possess little power of localisation, hence a limited irritation may canse diffused pain, or a general irritation may be referred to one spot, as we in affections of the periostenm and serous membranes. The degree of localisation largely depends on the number of nerves distributed to a part.

Though there thus appears to be no doubt as to the sensibility of the dara mater, the question is whether in all cases of intracranial pain it is possible to show the existence of such conditions as are calculated to cause irritation of the nerves of the dura mater, viz. abnormal tension or inflammatory action. If it should appear that there are forms of intracranial disease associated with pain in the head, complained of spontaneously or capable of being made manifest, in which, so far as can be judged, no conditions exist likely to cause irritation of the nerves of the dura mater, we have to inquire whether it is possible to refer the pain otherwise than to the cerebral tissue itself.

Besides the dura mater there are two other membranes investing the brain, the arachnoid and pia mater, or rather one membrane with two lajers more or less closely united, the arachno-pia or, shortly, the pia mater. Pathologically, at least, we can scircely regard them as separable and distinct.

The pia mater is generally regarded as insensitive. Nerves, however, have been traced to it by Luschka, Bochdalek, and others. These are regarded by Kölliker as belonging exclusively to the blood-vessels, and derived, like other vaso-motor 
nerves, from the sympathetic. As to the branches from the sympathetic there can be no doubt, but the existence of others which have been traced from the roots of some of the cranial nerves, as well as from the crus and medulla, is very questionable.

No indications of pain are, so far as can be judged, obsertable on electrical irritation of the pia mater in the lower unimals. The calibre of the blood-vessels alone seems altered, as on irritation of vaso-motor nerves in general. But this does not exclude the possibility that the pia mater may, like other tissues supplied only by the sympathetic, ander certain abnormal conditions, such as inflammation, become the seat of pain, its organic sensibility, according to Bichat, being transformed into the sensibility of relation.

Bichat regarded the arachnoid as so sensitive.

And that the pia mater, though only possessing vascular nerves, may become painfully sensitive is supported by the phenomena of arteritis and phlebitis, and by the acute pain experienced on traction on the ligature of an inflamed and ulcerating vessel. The sensibility of the vascular walls has received little attention, but they were regarded as sensitive by Bichat and others. It is not unlikely that hyperasthesia of the vascular walls in inflamed parts may be partly the cause of the peculiar throbbing pain synchronous with the pulse, which is then experienced. And to tonic spasm or cramp of the vascular walls, Helmholtz, as is well known, attributes the acute pain of hemicrania, which he has studied minutely in his own case.

It is probable, therefore, that the pia mater, though apparently insensitive, may become the seat of pain under conditions of disease, and this view is supported by a consideration of certain forms of cerebral disense, in which, without evident affection of the dura mater, pain is spontaneously complained of or may be elicited by percussion.

If these considerations are justified, an analysis of the phenomena of cerebral disease ought to show that those forms of disease are especially associated with headache in which there is undue, and more particularly rapid, increase of the intracranial pressure, or in which there is inflammation of the 
dura mater, or of the pia mater primarily or secondary to encephalitis.

That those diseases are asanciated with intense headache in which there is abnormal increase of the intracranial pressure and thereby undue tension of the cerebral membranes is amply justified by clinical facts. And that undue tension alone is a sufficient cause of pain in the head is tolerably clear. For though in many cases tension is accompanied by inflammation, and is the result of the inflammatory process, yet this is not always 80, and there seems a distinct proportion between the intensity of the pain and the amount and suddenness of the tension. To this must be ascribed almost exclusivelv the intense headache which accompanies cerebral tumours. These are sometimes complicated with inflammation, but the pain may be excruciating in the absence of inflammatory action.

To abnormal tension we may also ascribe, in great measure at least, the pain which accompanies pachymeningitis interna, or hæmatoma of the dura mater, meningeal hæmorrhage, acute hypertrophy of the brain, rapid effusion into the ventricles without evident inflammatory indications, as in leptomeningitis infantum, rapidly increasing cerebral abscess, and generally rapid increase of the intracranial pressure, whether associated or not with inflammation of the membranes.

The tension on the membranes will naturally be produced wherever the distending cause may be situated; whether, therefore, it is central or superficial, but the membranes will suffer more partially if the disease is superficial than when it is central. Hence, besides the general symptoms of increased intracranial pressure, there may be more or less localisable pain of a specially severe character.

What amount of tension in excess of the normal intracranial pressure is necessary to cause pain in the head we have no data for estimating exactly. But it would appear that severe pain may result from a degree of tension which falls far short of exerting destructive pressure on the vital centres of the pons and medulla, or inducing obvious stasis in the retinal veins. It is doubtful whether mere engorgement of the cerebral vessels is sufficient to cause headache by tension, for the equally grent, if not greater, tension induced by 
powerful expiratory efforts, or long-continued muscular strain, does not commonly canse headache. The headache of 80called congestion of the brain is more probably a concomitant symptom of a general condition, the pathology of which is obscure, thau a mere mechanical result of vascular engorgement.

Next, as to inflummation. That diseases of the brain and its membranes of an inflammatory nature, whether simple or specific, are accompanied by excruciating pain before tension, resulting from the products of inflammation, can have appreciably increased is evident from the phenomena of meningitis, whether simple, tubercular, or syphilitic. There is no diffculty in accounting for the pain when the inflammation affects the dura mater. But acute pain may be felt, more or less referable to the seat of disease, in which the inflammatory action does not, as far as can be seen, implicate the dura mater, either primarily or secondarily. In such cases, unless we suppose some obscure or indirect irritation of the nerves of the dura mater, it is more reasonable to regard the pia mater as the seat of pain.

In tubercular meningitis, in which the pia mater is the primary seat of the disease, pain is frequently intense before the oscurrence of tension from inflammatory products, and without any-evident affection of the dura mater.

Also in syphilitio affections of the cortex pain in the head is a characteristic symptom, and this, too, in cases where the dura mater, cranium or pericranium is not obviously affected. The fact pointed out by Buzzard that attacks of syphilitic epilepsy are often preceded by localised headache, though not exclnding general affection of the meninges, may be taken to support the view that the pain and irritation of the cortex are intimately related to each other.

In encephalitis pain is frequently intense without any implication of the dura mater in the inflammatory action. In the cases classed under the general head of "ramollissement," Rostan regarded as a pathognomonic sign the fact that the patient, even when plunged in semi-stupor and hemiplegic, would, when asked, point with his free hand to the same side of the head as the seat of pain. 
It has frequently been observed that the nearer the disease approaches the cortex, the greater the indications of pain.

In the ganglia and medullary substance of the hemispheres it is not unusual to discover affections of an inflammatory nature, or surrounded by a zone of inflammation, which have run their whole course without pain. Thus cerebral abscess situated in certain parts of the hemispheres may be latent not only as regards cerebral symptoms but also as regards pain in the head, particularly when it forms slowly and merely replaces normal brain tissue without adding much to the volume of the cranial contents.

In ninety-nine cases of cerebral disease analysed by Mr. Callender there was not one accompanied with pain in the head when the ganglia alone were the seat of lesion. In ordinary central hemiplegia depending on effusions, \&c., into the ganglia, pain is rarely complained of spontaneously, and is not, so far as I have found, elicited by percussion. The sudden increase of pressure which results from a hæmorrhagio effusion ought, in accordance with the principles laid down, to canse acute pain in the head; but as consciousness is obscured at the same time by pressure, the pain cannot be said to exist, though it is frequently compleined of when consciousness returns, and before the abnormal tension has disappeared.

But apart from the indirect effects of pressure, there does not seam to be any pain associated with homorrhagic lesions in the contral ganglia or substance of the hemispheres.

It rather militates against the inflammatory nuture of the cortical affections in general paralysis, or at least points to some peculiarity-possibly the very chronic character of the process -that the subject of it appears to be free from headache. Crichton-Browne, however, finds that this freedom from headache is by no means constant, and that sometimes there are indications of excruciating pain in the head, a fact which strongly supports the view that it is a form of meningoencephalitis.

It would appear, therefore, that those diseases are especially painful which directly or indirectly affect the cerebral membranes, and that we have reasons for regarding the pia mater as well as the dura mater as the seat of the pain. Other facts 
to be mentioned below are, in my opinion, only explicable on this hypothesis.

Next, as regards the locality of the pain in cerebral disease. Though this frequently coincides exactly or approximately with the seat of lesion, a fact which is the best indication of the intracranial origin of the pain, yet the rule is by no means constant, and it would lead to serious error if the locality of the pain were taken by itself as a guide to the position of the lesion. The situation of the pain corresponds to the position of the lesion, more particularly in cases of cerebellar disease, or disesse in the posterior foses of the skull. In the vast majority of instances the pain is referred to the occipital region. In some cases, however, of cerebellar disease the pain is referred to the frontal region. As a general rule the less localisable the pain is, the greater the tendency to refer it to the frontal region. Why this is 80 we cannot satisfactorily explain. But that the frontal region is the lieu d'election in most cases of headache of sympathetic origin and in many cases of organic cerebral disease is undoubted. It is not difficult to account for the localisation of the pain in the occipital region in cases of cerebellar disease, particularly tumours, when we consider how the posterior fossu of the skull is cut off from the rest of the cranial cavity by the tentorium, and how therefore the tension is mainly concentrated in a comparatively closed spaco.

In affections of the cerebral hemispheres, thongh the pain is often correctly referred to that part of the cranium overlying the disease, yet this is less constant than in cerebellar disease. Often the pain is diffused, and if it is referred to one particular region this does not always correspond with the position of the lesion. In sixteen cases of meningitis Andral found five in which the headache was vaguely referred to the whole head. In eleven, the pain was referred to a limited region. In six of these only did the situation of the pain correspond to the situation of the disease. In the other five there was no correspondence between the two. In one the disease was in the left hemisphere, and the pain was referred to the forehead. In the second the pain was referred to the left side of the head, and yet the meningitis was general. In the third the

VOL. 1. 
pain was referred to the temples, and the meningitis was basilar. In the other two the disease was in the ventricles, and the pain was referred to the forehead. In the five cases, however, in which the pain was not localised, the disease was also general, except in one in which the lesion was restricted mainly to the frontal regions. It is rare, therefore, to have localised headache when the disease is not also cincumscribed. What is true of meningitis holds also in reference to encephalitis and other organic cerebral diseases in which pain is complained of.

The locality of the pain, therefore, even when it is circumscribed, cannot be taken as a faithful index of the situation of the disease, and we have to rely mainly on the nature of the functional disturbances for our regional diagnosis. Here, however, we have to encounter the difficulty of determining in a given case whether the lesion is actually situated in the part or centre the functions of which are particularly affected, or situated at some distance, acts only indirectly by pressure or otherrise on those parts, the functional derangements of which are the chief or only obvious symptoms. This is a question which it would be of the highest importance to be able to answer decisively, more particularly in view of local treatment or surgical interference. If trephining for cerebral disease other than in cases where there are clear external indications should become more generally practised, every indication will be of value which may serve to fix the exact position of the lesion.

It is with this object that I desire to call the attention of physicians to percussion of the sknll in cerebral disease, even when the patient makes no spontaneous complaint of pain in the head, as an aid in fixing the locality of cerebral lesions, more particularly those of the cortex. I have frequently been struck with the existence of pain on percussion over the cranial region, corresponding to the situation in which from other facts I had been led to localise some lesion, or the intensification of the pain spontaneously complained of in some particular spot where it had been more or less vaguely felt. Theoretically nothing seems better calculated to intensify pain, or elicit it, in hyperæsthetic parts, than the jarring which is caused by smart percussion over the region affected. This I have found to be the case even when the lesion was 
evidently of an embolic nature, a fact which appears capable of explanation only, or at least most probably, on the view that the pia mater is the seat of pain, and rendered hyperwsthetic by the inflammatory zone described by Hasse as surrounding the necrosed part.

The following are a few examples illustrative of the foregoing remarks.

In a female patient under the care of Dr. Clonston in the Morningside Asylum, who had been admitted eight months previously with symptoms of excitement, aphasia and paresis of the right arm and right side of the face, I found that percussion in the left temporal region over the anterior inferior angle of the parietal bone caused acute pain. The patient did not complain of pain in the head spontaneously, and only in this region was percussion painful. Dr. Clouston informs me that this tenderness on percussion continued for twelve months afterwards. The aphasia still remains.

This patient is subject to angina poctoris, the heart is hypertrophied, and the sounds are muffled, bat there is no distinct murmur. Though the nature of the lesion is not quite certain, the facts are in favour of embolism.

The pain in this case was of long duration, but occasionally it is very evanescent.

Thus in a case of right brachio-facial paralysis, with slight aphasia of two days' duration, which came under my care some months ago at King's College Hospital, there was pain on percussion in a similar position in the left temporal region. The pain, however, had disappeared a few days subsequently, when he was admitted into the hospital. The patient quite recovered from his attack in a few weeks, but returned shortly after being discharged with a similar affection of the left arm and left side of the face, but without aphasia. There was no pain on percussion this time, and the symptoms again rapidly disappeared.

In January last a female patient came to me at the Hospital for Epilepsy and Paralysis, suffering from paresis of the right hand and right side of the face. Sensation and electrical reactions were normal. There was no aphasia. She stated, that a few days previously while in the act of pouring out tea 
she suddenly dropped the teapot, and found she had lost the nse of her right hand. The history as well as the existence of distinct presystolic murmur clearly pointed to embolism. She complained of no pain in the head. But on percussion a painful spot was found just above the left ear, a region corresponding to the position of the lower extremity of the ascending parietal convolution, lesion in which would readily explain the symptoms. The pain gradually diminished, and disappeared on recovery of movement in the course of six weeks.

In another case which I saw at the same hospital the patient had been suddenly seized about a year previously with paralysis of the right leg, which gradually extended to the right arm. The right arm and leg were paretic, but the face was unaffected. Sensibility and electrical reactions were normal. He stated the attack had come on suddenly, with partial loss of consciousness and pain in the left side of the head, during sexual intercourse. The pain in the head had, according to the patient's account, quite disappeared, but, on percussion of the skull, pain was elicited above the left parietal eminence close to the middle line. This would correspond with a lesion affecting the posterior and upper border of the fissure of Rolando, which would account for the symptoms. The lesion was probably superficial hæmorrhage.

Last May I was consulted in reference to a patient who, four months previously while playing at cards, had been suddenly seized with speechlessness and slight paresis of the right arm. After threo days apeech had returned, but was rather unintelligible, and he did not seem to understand what was said to him. He recovered to some extent, but had a similar attack while playing at bowls three months after the first. This time he complained of pain in the head, and he gradually passed into the state in which I found him.

He had no paralysis. He could articulate fairly well, and read ; but he seemed utterly unable to comprehend what was said to him, except such simple things as "stand," "sit down," drc. He looked u'terly bewildered when asked such questions as, Whether he had any pain in the head? He could understand gestures, and could make out the meaning of what was written for him on a slate which he kept for the purpose. He had 
long been deaf in the left ear, but he could hear distinotly with the right. In short, the symptoms were typical of "surditas verbalis," or "word-deafness." He had a systolic murmur, both at apex and base, and a well-marked diastolic murmur, with the pulse of aortic regurgitation.

$\mathrm{He}$ complained of no pain in the head, but on percussion pain was elicited in the left parietal region below the parietal eminence. The symptoms in this case would be accounted for by a lesion affecting the superior temporo-sphenoidal convolution and margins of the fissure of Sylvius, and the situation of the painful area would correspond with a lesion 80 situated. The case therefore is like one reported by Wernicke, in which a lesion was found in this part of the cortex after death.

The lesion was probably embolic, as the age of the patient (38), the absence of syphilis, the mode of onset, and the pre sence of extensive valvular disease would indicate.

This patient recently died with cardiao symptoms, but unfortunately a post-mortem examination could not be made. Dr. Parsons of Liverpool, under whose care the patient was, informs me that the pain in the neighbourhood of the left parietal eminence could always be elicited up to two months before death.

I had under my care at King's College Hospital a patient whose chief symptoms were a staggering and uncertain gait, which had come on after a fit in which he had temporarily lost consciousness. The staggering was especially observable when the patient was made to wheel to the right, less so when he wheeled to the left. The symptoms gradually disappeared, the last sign of the unsteadiness being the inebility to hop round on the right foot, while he could do this easily on the left.

In this case there was marked pain on percussion in the right occipital region. From this, as well as from the peculiar symptoms, I diagnosed lesion of the right cerebellar lobe, probably due to cerebellar hømorrhage of limited extent, which became absorbed. The pain in the right occipital region disappeared with recovery from the other symptoms.

I have at present under my care a patient who staggers much when walking, and who cannot stand when the foet 
are placed close together. On attempting to do this he invariably falls backwards. The symptoms are not intensified appreciably on closure of the eyes, and there is no real ataxy, nor are any of the characteristic symptoms of ataxy present. There is also complete deafness in the left ear, partial paralysis of motion and anmsthesia on the left side of the face, and loss of tactile sensibility and taste on left anterior two-thirds of the tongue. He has occasional pain in the head in the occipital region, with nausea and feeling of sickness, but there is no optio neuritis, though perhaps the veins are somewhat larger than normal. The symptoms point strongly to cerebellar tumour, and pressure on the fifth and seventh cranial nerves of the left side.

In this patient percussion over the left superior curved line of the occiput canses very marked intensification of the headache which he suffers from, but not continually, in the occipital region. The symptoms and the situation of the pain are thus mutually confirmatory of lesion, probably tumour, in the left cerebellar lobe.

In syphilitic lesions of the cortex pain is, as has boen stated, often spontaneously complained of, and very frequently in a region corresponding to the position of the disease. This, however, is not always so. If it exists, it is intensified by percussion, but percussion may serve to elicit it when it is not otherwise perceptible.

Dr. Robertson has recorded a 'case ('Brit. Med. Journal,' May 18, 1878) of syphilitic epilepsy. affecting the right side of the body, beginning chiefly in the right hand, and usually without loss of consciousness. The attacks were followed by a greater or less degree of paralysis of the parts affected by the convulsive spasms.

The patient did not spontaneously complain of pain in the head, but on percussion of the skull pain was elicited in a circular area two inches in diameter, the lower margin of which was two inches above the highest point of the ear. As Dr. Robertson remarks, with reason, it is probable that the lesion did not implicate the skull or dara mater, as in such conditions the pain is usually distinct enough, and often severe, epecially at night, and not requiring artificial elicitation. 
The locality of the pain would conflrm the diagnosis of a syphilitic lesion of the cortex in the convolutions bounding the fissure of Rolando.

In a case which I had under my care two years ago there was a syphilitic history, and the patient was subject to repeatedattacks of spesm of the right leg, sometimes occurring during walking and causing denger of falling. Frequently the right arm was implicated, and sometimes the right side of the face also. The patient never lost consciousness in his attacks.

He had no pain in the head, but percussion of the skull caused uneasiness when applied over the posterior superior angle of the left parietal bone close to the middle line. This would confirm the diagnosis of a cortical lesion affecting espocially the superior parietal lobule. The symptoms were greatly ameliorated under the influence of iodide of potassium, 80 long as the patient remained under my care, but I can say nothing as to the subsequent progress of the case, as I have lost sight of him.

Last November I was consulted by a patient, aged 25, who had paresis of the left arm. Two years before he had contracted syphilis, and some months before he consalted me he had woke up one morning with numbness and loss of power in the left hand and arm. This gradually diminished, when, three weeks after, he was suddenly seized while walking in the street with paralysis of the left arm and slight staggering. He did not lose his spech, though, as he said, he experienced some difficulty in articalation. He had a headache at the time, and his thoughts were somewhat confused. When I saw him the only indications of symptoms remaining were slight paralysis of motion of the left hand and arm.

There was no cardiac disease, and he had no pain in the head. On percussion of the skull, pain was elicited in the middle of the right parietal region, just anterior to the parietal eminence.

Under the influence of iodide of potassium the paresis disappeared in the course of six weeks, and the pain on percussion of the right parietal region was no longer felt.

The locality of the pain, taken with the symptoms, indicated lesion in the escending parietal convolution, and the rapid 
disappearance of the symptoms under specific treatment justified the diagnosis of a syphilitic affection of the cortex.

I have at present under my care a patient, a girl aged 8 years, whose history and symptoms indicate that a tubercular affection of the cortex may exist without causing headache, which, howerer, may be elicited by percussion of the skull.

This patient belongs to a family, two of which have died in infancy with all the symptoms of tubercular meningitis. A younger sister has a very large head with bulging forehead, and the usual appearances of the strumous diathesis.

She has been subject to recurrent fits, in which the prominent symptoms, described by her mother, are a remarkable turning of her head and eyes to the left. She does not fall, and there does not appear to be complete loss of consciousness during her fit. The left arm is paretic, the grasp feeble, and there is some rigidity of the flexors of left hand. The face is slightly drawn to the right when she grins. The left leg seems as strong as the right, though her mother says it was at one time distinctly weak. Sensation and electric contractibility are normal. She has usually no pain in the head or nausea, though at the period of attacks she has some pain in the head and a tendency to sickness. The optic discs are sharp and present no abnormal appearance.

On percussion of the skull she indicates a spot in which pain is felt, situated about an inch and a half from the sagittal suture in a line drawn vertically upwards from the external anditory meatus.

The symptoms in this case, taken with the general history, seem to point to a tubercular affection of the cortex; and they would be capable of explanation by a lesion situated in the upper half of the ascending frontal convolution and junction of the upper and middle frontal.

The position of the percussion pain would correspond very closely with a lesion so situated.

I freely admit that in the absence of post-mortem. confirmation these various facts do not altogether satisfactorily establish a correspondence between the locality of the percussion pain and the cerebral lesion. I have not, however, had 
IN CONNECTION WITH CEREBRAL DISEASE. 483

an opportunity of post-mortem examination in cases which $I$ have carefully examined in this way.

But it appears to me a fact of very great significance and corroboration of regional diagnosis otherwise arrived at, that percussion of the skull so often elicited pain over the region where the lesion was localised. If the correspondence cannot as yet be regarded as conclusively proved, yet I think the facts warrant a more general adoption of percussion of the head in the localisation of cerebral disease with the view of testing its value over a much wider range of clinical and pathological observation than can possibly occur to any one individual. 